\title{
On Conditions for Denominator Ideals to Diffuse and Conditions for Elements to Be Exclusive in Anti-Integral Extensions
}

\author{
Susumu OdA* AND Ken-ICHI Yoshida**
}

Let $R$ be a Noetherian integral domain and let $\alpha$ be an element in an algebraic extesnision field of the quotient field of $R$. Our objective is to show that a generalized denominator ideal $I_{[\alpha]}$ satisfies $I_{[\alpha]} R[\alpha]=R[\alpha]$ if and only if $I_{[\alpha]}+I_{\left[\alpha^{-1}\right]}=R$ if and only if either $\alpha$ or $\alpha^{-1}$ is integral over $R_{p}$ for each $p \in \operatorname{Spec}(R)$, provided that $\alpha$. is an anti-integral element over $R$. The other is to show that for the canonical map $\phi: \operatorname{Spec}(R[\alpha]) \rightarrow \operatorname{Spec}(R), \operatorname{Dp}_{1}(R)$ is contained in the image of $\phi$ if and only if $\alpha$ is exclusive. The former result is closely concerned to [KY1] and [OY3], and the latter result is related to [OY2].

\section{Notation and Convensions}

Throughout this paper, we use the following notation unless otherwise specified:

Let $R$ be a Noetherian domain (which is commutative and has a unit), let $R[X]$ be a polynomial ring, let. $\alpha$ be an element of an algebraic extension field of the quotient field $K$ of $R$ and let $\pi: R[X] \rightarrow R[\alpha]$ be the $R$-algebra homomorphism sending $X$ to $\alpha$. Let $\varphi_{\alpha}(X)$ be the monic minimal polynomial of $\alpha$ over $K$ with $\operatorname{deg} \varphi_{\alpha}(X)=d$ and write $\varphi_{\alpha}(X)=X^{d}+\eta_{1} X^{d-1}+\cdots+\eta_{d}$. Then $\eta_{i} \in K(1 \leq i \leq d)$ are uniquely determined by $\alpha$. Put $d=[K(\alpha): K]$, $I_{\eta_{i}}:=R: R \eta_{i}$ and $I_{[\alpha]}:=\bigcap_{i=1}^{d} I_{\eta_{i}}$. If $\operatorname{Ker}(\pi)=I_{[\alpha]} \varphi_{\alpha}(X) R[X]$, we say that $\alpha$ is anti-integral over $R$ (cf. [OSY]). Put $J_{[\alpha]}:=I_{[\alpha]}\left(1, \eta_{1}, \ldots, \eta_{d}\right)$. For $\beta \in K$, we put $J_{\beta}:=I_{\beta}(1, \beta)$. Then $J_{[\alpha]}=\mathrm{c}\left(I_{[\alpha]} \varphi_{\alpha}(X)\right)$, where $\mathrm{c}()$ denotes the ideal generated by the coefficients of the polynomials in () , that is, the content ideal of ( ). If $J_{[\alpha]} \nsubseteq p$ for all $p \in \operatorname{Dp}_{1}(R):=\left\{p \in \operatorname{Spec}(R) \mid \operatorname{depth} R_{p}=1\right\}$, the element $\alpha$ is called a super-primitive element over $R$. A super-primitive element over $R$ is anti-integral over $R$ ( $c f$.[OSY,Theorem 1.12]). It is also known that any algebraic element over a Krull domain $R$ is super-primitive over $R$ ( $c f$. [OSY, Theorem 1.13]), and hence $\alpha$ is anti-integral over $R$. We also note here that $I_{[\alpha]}=R \Leftrightarrow R[\alpha]$ is integral over $R$ and that $J_{[\alpha]}=R \Leftrightarrow R[\alpha]$ is flat over $R$, provided that $\alpha$ is anti-integral over $R$

\section{Received May 26, 1998.}

1991 Mathematics Subject Classification. Primary 13C20, Secondary 13 F99.

* Matsusaka Commercial High School Toyohara, Matsusaka, Mie 515-0205 Japan

e-mail :

** Department of Applied Math. Okayama University of Science Ridai-cho, Okayama 700-0005

Japan

e-mail : 
Our general reference for unexplained terminology is $[\mathrm{M}]$.

\section{$\S 1$. Conditions for generalized denominator ideals to diffuse in anti-integral extensions}

We start with the following proposition. The implication (i) $\Rightarrow$ (ii) is seen in [OY3,Proposition 7], but we give a proof for convenience.

Proposition 1.1. Assume that $\alpha$ is an anti-integral element over $R$. Then the following statements are equivalent:

(i) $I_{[\alpha]} R[\alpha]=R[\alpha]$;

(ii) $\eta_{d} \in R[\alpha], I_{[\alpha]}=I_{\eta_{d}}$ and $J_{\eta_{d}}=R$.

Proof. If $I_{[\alpha]}=R$, then the implications (i) $\Leftrightarrow$ (ii) are trivially valid because $\eta_{d} \in R$ by the definition of $I_{[\alpha]}$. So we may assume that $I_{[\alpha]} \neq R$.

(i) $\Rightarrow$ (ii) : Since $\eta_{1}, \ldots, \eta_{d} \in I_{[\alpha]}^{-1} R[\alpha]=I_{[\alpha]}^{-1} I_{[\alpha]} R[\alpha] \subseteq R[\alpha]$, we have $\eta_{d} \in R[\alpha]$. Put $C:=R\left[\eta_{1}, \ldots, \eta_{d}\right]$. Then $R[\alpha]$ is a free $C$-module and $R[\alpha]$ is integral over $C$. So $I_{[\alpha]} R[\alpha]=R[\alpha]$ yields that $I_{[\alpha]} C=C$. Thus $C$ is flat over $R$. Since $R[\alpha]$ is flat over $C, R[\alpha]$ is flat over $R$, and hence $J_{[\alpha]}=R$. Now we shall show that $I_{[\alpha]}=I_{\eta_{d}}$. It is easy to see that $I_{[\alpha]} \subseteq I_{\eta_{d}}$. Take $p \in \operatorname{Spec}(R)$ with $p \supseteq I_{[\alpha]}$. Since $I_{[\alpha]} R[\alpha]=R[\alpha]$, we have $1=a_{0}+a_{1} \alpha+$ $\cdots+a_{n} \alpha^{n}$, where $a_{i} \in I_{[\alpha]} \subseteq p$. Since $1-a_{0}$ is a unit in $R_{p}, \alpha^{-1}$ is integral over $R$. Since $\alpha$ is anti-integral over $R, \alpha^{-1}$ is also anti-integral over $R$. Thus $\varphi_{\alpha^{-1}}(X)=X^{d}+\left(\eta_{d}^{-1} \eta_{d-1}\right) X^{d-1}+\cdots+\left(\eta_{d}^{-1} \eta_{1}\right) X+\eta_{d}^{-1} \in R_{p}[X]$. So there exists $x \in R_{p}$ such that $\eta_{d}=1 / x, \eta_{i}=y_{i} / x$ with some $y_{i} \in R_{p}$. Therefore we have $\left(I_{[\alpha]}\right)_{p}=x R_{p}=\left(I_{\eta_{d}}\right)_{p}$. Since $p$ is arbitrary, we conclude that $I_{[\alpha]}=I_{\eta_{d}}$. Next we shall show that $J_{\eta_{d}}=I_{\eta_{d}}\left(1, \eta_{d}\right)=R$. Suppose that $I_{\eta_{d}}\left(1, \eta_{d}\right) \subseteq p$ for some $p \in \operatorname{Spec}(R)$. Then $I_{[\alpha]} \subseteq p$. Thus by using the above notation, we have $\left(I_{\eta_{d}}\left(1, \eta_{d}\right)\right)_{p}=x(1,1 / x) R_{p}=R_{p}$, which contradicts the assumption $I_{\eta_{d}}\left(1, \eta_{d}\right) \subseteq p$. Therefore we conclude that $J_{\eta_{d}}=R$.

(ii) $\Rightarrow$ (i) follows the implications : $R[\alpha] \supseteq I_{[\alpha]} R[\alpha] \supseteq I_{[\alpha]}\left(1, \eta_{d}\right)=I_{\eta_{d}}\left(1, \eta_{d}\right)$ $=R \ni 1$, and hence $R[\alpha] \supseteq I_{[\alpha]} R[\alpha] \supseteq R[\alpha]$.

Corollary 1.2. Assume that $\alpha$ is an anti-integral element over $R$. If $I_{[\alpha]} R[\alpha]=R[\alpha]$, then $R[\alpha] \cap K=R\left[\eta_{d}\right]$.

Proof. Put $C:=R\left[\eta_{1}, \ldots, \eta_{d}\right]$. Then by the proof of Proposition 1.1, we have $C \subseteq R[\alpha]$. Since $R[\alpha]$ is a free $C$-module $C+C \alpha+\cdots+C \alpha^{d-1}$, we conclude that $R[\alpha] \cap K=C$. Besides, as in the proof of Proposition 1 , if $I_{[\alpha]} \subseteq p$, then there exists $x$ in $R_{p}$ such that $\eta_{d}=1 / x$ and $\eta_{i}=y_{i} / x \in R_{p}\left[\eta_{d}\right]$ for some $y_{i} \in R_{p}$. If $I_{[\alpha]} \nsubseteq p$, then $C_{p}=R_{p}=R_{p}\left[\eta_{d}\right]$. Therefore we have $C=R\left[\eta_{d}\right]$.

LEMMA 1.3. $I_{\left[\alpha^{-1}\right]}=\eta_{d} I_{[\alpha]}$.

Proof. Since $\varphi_{\alpha^{-1}}(X)=X^{d}+\left(\eta_{d}^{-1} \eta_{d-1}\right) X^{d-1}+\cdots+\left(\eta_{d}^{-1} \eta_{1}\right) X+\eta_{d}^{-1}$, we have $I_{\left[\alpha^{-1}\right]}=\bigcap_{i=1}^{d-1} I_{\eta_{d}^{-1} \eta_{i}} \cap I_{\eta_{d}^{-1}}$. Take $x=\eta_{d} y \in \eta_{d} I_{[\alpha]}$ with $y \in I_{[\alpha]}$. Then $x \eta_{d}^{-1} \eta_{i}=y \eta_{i} \in R$ and hence $x \eta_{d}^{-1}=y \in R$. Thus $I_{\left[\alpha^{-1}\right]} \supseteq \eta_{d} I_{[\alpha]}$. Conversely, 
take $x \in I_{\left[\alpha^{-1}\right]}$. Then $x \eta_{d}^{-1}=y \in R, y \eta_{d}=x \in R$ and $x \eta_{d}^{-1} \eta_{i}=y \eta_{i} \in R$. hence $y \in I_{[\alpha]}$. Therefore we have $I_{\left[\alpha^{-1}\right]}=\eta_{d} I_{[\alpha]}$.

REMARK 1.4. (i) An element $\alpha$ is anti-integral over $R$ if and only if so is $\alpha^{-1}$ (cf. [KY]).

(ii) An element $\alpha$ is super-primitive over $R$ if and only if so is $\alpha^{-1}$. Indeed, the minimal monic polynomial $\varphi_{\alpha^{-1}}$ of $\alpha^{-1}$ is $X^{d}+\left(\eta_{d-1} / \eta_{d}\right) X^{d-1}+\cdots+$ $\left(\eta_{1} / \eta_{d}\right) X+\left(1 / \eta_{d}\right)$. Hence we have

$$
\begin{aligned}
& J_{\left[\alpha^{-1}\right]} \\
& =I_{\left[\alpha^{-1}\right]}\left(1, \eta_{d-1} / \eta_{d}, \ldots, \eta_{1} / \eta_{d}\right) \\
& =\eta_{d} I_{[\alpha]}\left(1, \eta_{d-1} / \eta_{d}, \ldots, \eta_{1} / \eta_{d}\right) \\
& =I_{[\alpha]}\left(1, \eta_{1}, \ldots, \eta_{d}\right)=J_{[\alpha]},
\end{aligned}
$$

here we use Lemma 1.3. So our assertion follows from the definition of superprimitiveness.

THEOREM 1.5. Assume that $\alpha$ is an anti-integral element over $R$ and that $\eta_{d} \in R[\alpha]$. The following statements are equivalent:

(i) $I_{[\alpha]} R[\alpha]=R[\alpha]$;

(ii) $I_{[\alpha]}+I_{[\alpha-1]}=R$;

(iii) either $\alpha$ or $\alpha^{-1}$ is integral over $R_{p}$ for each $p \in \operatorname{Spec}(R)$.

Proof. (i) $\Rightarrow$ (ii) : We have $\eta_{d} \in R[\alpha], I_{[\alpha]}=I_{\eta_{d}}$ and $J_{\eta_{d}}=R$ by Proposition 1.1. So we obtain $R=J_{\eta_{d}}=I_{\eta_{d}}\left(1, \eta_{d}\right)=I_{[\alpha]}\left(1, \eta_{d}\right)=I_{[\alpha]}+\eta_{d} I_{[\alpha]}=$ $I_{[\alpha]}+I_{\left[\alpha^{-1}\right]}$ (cf. Lemma 1.3).

(ii) $\Rightarrow$ (i): We have $R=I_{[\alpha]}+I_{\left[\alpha^{-1}\right]}=I_{[\alpha]}+\eta_{d} I_{[\alpha]}=I_{[\alpha]}\left(1, \eta_{d}\right)$. Thus $R[\alpha]=I_{[\alpha]}\left(1, \eta_{d}\right) R[\alpha]=I_{[\alpha]} R[\alpha]+I_{[\alpha]} \eta_{d} R[\alpha] \subseteq I_{[\alpha]} R[\alpha]$ because $\eta_{d} \in R[\alpha]$. The converse inclusion is obvious. Thus $I_{[\alpha]} R[\alpha]=R[\alpha]$.

(ii) $\Rightarrow$ (iii) : Take $p \in \operatorname{Spec}(R)$. Then $I_{[\alpha]} \nsubseteq p$ or $I_{\left[\alpha^{-1}\right]} \nsubseteq p$ because $I_{[\alpha]}+$ $I_{\left[\alpha^{-1}\right]}=R$. If $I_{[\alpha]} \nsubseteq p$, we have $\eta_{1}, \ldots, \eta_{d} \in R_{p}$. Hence $\alpha^{d}+\eta_{1} \alpha^{d-1}+\cdots+\eta_{d}=0$, which means that $\alpha$ is integral over $R_{p}$. Next, we assume that $I_{\left[\alpha^{-1}\right]} \nsubseteq p$. The $\eta_{d}^{-1} \eta_{1}, \ldots, \eta_{d}^{-1} \eta_{d-1}, \eta_{d}^{-1} \in R_{p}$. Thus $\left(\alpha^{-1}\right)^{d}+\left(\eta_{d}^{-1} \eta_{d-1}\right) \alpha^{d-1}+\cdots+\eta_{d}^{-1} \eta_{1} \alpha+$ $\eta_{d}^{-1}=0$, which means that $\alpha^{-1}$ is integral over $R_{p}$.

(iii) $\Rightarrow$ (ii) : Suppose that there exists a prime ideal $p$ of $R$ such that $I_{[\alpha]}+I_{\left[\alpha^{-1}\right]} \subseteq p$. Note that $\alpha$ or $\alpha^{-1}$ is integral over $R_{p}$. Assume that $\alpha$ is integral over $R_{p}$. Then $\alpha$ satisfies a monic relation of degree $d$ over $R_{p}$ because $\alpha$ is anti-integral over $R_{p}$. But since $\varphi_{\alpha}(X)=X^{d}+\eta_{1} X^{d-1}+\cdots+\eta_{d} \in R_{p}[X]$, we have $I_{[\alpha]} \nsubseteq p$, a contradiction. Similarly we come to a contradiction when we assume $\alpha^{-1}$ is integral over $R_{p}$.

Proposition 1.6. Assume that $\alpha$ is an anti-integral element of degree $d$ over $R$. If $\eta_{d} \in R[\alpha]$, then $\sqrt{I_{[\alpha]} R[\alpha] \cap R}=\sqrt{I_{[\alpha]}+I_{\left[\alpha^{-1}\right]}}$.

Proof. Take $p \in \operatorname{Spec}(R)$. Then $I_{[\alpha]} R[\alpha] \cap R \nsubseteq p \Leftrightarrow I_{[\alpha]} R_{p}[\alpha]=R_{p}[\alpha] \Leftrightarrow$ $\left(I_{[\alpha]}+I_{\left[\alpha^{-1}\right]}\right)_{p}=R_{p} \Leftrightarrow I_{[\alpha]}+I_{\left[\alpha^{-1}\right]} \nsubseteq p$. Thus we come to our conclusion by Theorem 1.5. 


\section{$\S 2$. Conditions for anti-integral elements to be exclusive.}

Recall first that an algebraic element $\alpha$ over $R$ is called to be exclusive if $R[\alpha] \cap K=R$. Put $\tilde{J}_{[\alpha]}:=I_{[\alpha]}\left(1, \eta_{1}, \ldots, \eta_{d-1}\right)$, an ideal of $R$.

REMARK 2.1. Assume that $R$ contains a field $k$. Let $x$ be an indeterminate and put $S:=R \otimes_{k} k(x)$. Then $S$ contains the infinte field $k(x)$. Put $I_{[\alpha]}^{S}:=$ $\bigcap_{i=1}^{d}\left(S: S \eta_{i}\right), J_{[\alpha]}^{S}:=I_{[\alpha]}^{S}\left(1, \eta_{1}, \ldots, \eta_{d}\right) S$ and $\tilde{J}_{[\alpha]}^{S}:=I_{[\alpha]}^{S}\left(1, \eta_{1}, \ldots, \eta_{d-1}\right) S$. Since $S$ is faithfully flat over $R, I_{[\alpha]}^{S}=I_{[\alpha]} S, J_{[\alpha]}^{S}=J_{[\alpha]} S$ and $\tilde{J}_{[\alpha]}^{S}=\tilde{J}_{[\alpha]} S$. So if $\alpha$ is supcr-primitive (resp. anti-integral) over $R$, then so is $\alpha$ over $S$. Moreover $\alpha$ is exclusive over $S$ if and only if so is $\alpha$ over $R ; \bigcap_{i=1}^{d-1} I_{\eta_{i}} \subseteq I_{\eta_{d}}$ if and only if $\bigcap_{i=1}^{d-1} I_{\eta_{i}}^{S} \subseteq I_{\eta_{d}}^{S}$; $\operatorname{grade}\left(\tilde{J}_{[\alpha]}\right)>1$ if and only if $\operatorname{grade}\left(\tilde{J}_{[\alpha]}^{S}\right)>1$; and $\alpha$ is exclusive over $S$, i.e., $S[\alpha] \cap K(x)=S[\alpha]$ if and only if $\alpha$ is exclusive over $R$, i.e., $R[\alpha] \cap K=R$.

Lemma .2.2( cf. [OY2,Theorem 5]) Assume that $\alpha$ is super-primitive over R. Then the following statements (i) and (ii) are equivalent :

(i) $\bigcap_{i=1}^{d-1} I_{\eta_{i}} \subseteq I_{\eta_{d}}$;

(ii) $\operatorname{grade}\left(\tilde{J}_{[\alpha]}\right)>1$ or $\tilde{J}_{[\alpha]}=R$.

Furthermore if $R$ contains a field, then the following (iii) is equivalent to (i)

(iii) $\alpha$ is exclusive over $R$.

Proof. (i) $\Leftrightarrow$ (ii) follows from [OY2,Lemma 3].

Next assume that $R$ contains a field. We may assume that $R$ contains an infinite field by Remark 2.1. Hence our conclusion (ii) $\Leftrightarrow$ (iii) follows from [OY2, Theorem 5].

REMARK 2.3. Assume that $R$ contains a field.

(i) When $\alpha$ is a super-primitive element over $R, \alpha$ is exclusive over $R$ if and only if $\operatorname{grade}\left(\tilde{J}_{[\alpha]}\right)>1$ by Lemma 2.2 .

(ii) $\tilde{J}_{\left[\alpha^{-1}\right]}=I_{[\alpha]}\left(\eta_{1}, \ldots, \eta_{d}\right)$. This follows from the similar argument of Remark 1.4(ii).

(iii) By Remark 1.4, $\alpha$ is super-primitive over $R$ if and only if so is $\alpha^{-1}$. So by (i) and (ii) above, we have $\alpha^{-1}$ is exclusive over $R$ if and only if $\operatorname{grade}\left(I_{[\alpha]}\left(\eta_{1}, \ldots, \eta_{d}\right)\right)>1$.

(iv) If grade $\left(I_{[\alpha]}\left(\eta_{1}, \ldots, \eta_{d-1}\right)\right)>1$, then both $\alpha$ and $\alpha^{-1}$ are exclusive over $R$ by (i), (ii) and (iii).

Proposition 2.4. Assume that $\alpha$ is an anti-integral element of degree $d$ over $R$. If $I_{[\alpha]} R[\alpha]_{P}=R[\alpha]_{P}$ for every $P \in \mathrm{Dp}_{1}(R[\alpha])$, then $R[\alpha] \cap K=$ $R\left[\eta_{1}, \ldots, \eta_{d}\right]$.

Proof. It follows that $R[\alpha]_{P} \supseteq I_{[\alpha]}^{-1} I_{[\alpha]} R[\alpha]_{P}=I_{[\alpha]}^{-1} R[\alpha]_{P} \supseteq$ $I_{[\alpha]}^{-1} \ni \eta_{1}, \ldots, \eta_{d}$ because $\bigcap_{P \in \mathrm{D}_{\mathrm{p}_{1}}(R)} R[\alpha]_{P}=R[\alpha]$. Thus $R[\alpha] \cap K \supseteq$ $R\left[\eta_{1}, \ldots, \eta_{d}\right]=: C, \alpha$ is integral over $C$ and $\alpha$ is anti-integral over $C$. Since 
$K(C)=K$ and $[K(\alpha): K]=d, R[\alpha]$ is a free $C$-module $C+C \alpha+\cdots+C \alpha^{d-1}$. Hence $R[\alpha] \cap K=C$, as was to be shown.

Theorem 2.5. Assume that $\alpha$ is a super-primitive element of degree $d$ over $R$ and that $R$ contains a field. Let $\phi: \operatorname{Spec}(R[\alpha]) \rightarrow \operatorname{Spec}(R)$ be the canonical map induced from the inclusion $R \subseteq R[\alpha]$. Then the following statements are equivalent:

(i) $\mathrm{Dp}_{1}(R)$ is contained in the image of $\phi$, i.e., $\operatorname{Im}(\phi) \supseteq \mathrm{Dp}_{1}(R)$;

(ii) $\alpha$ is exclusive over $R$.

Proof. Im $(\phi) \supseteq \mathrm{Dp}_{1}(R)$ if and only if $\tilde{J}_{[\alpha] p}=J_{[\alpha] p}=R_{p}$ for every $p \in$ $\mathrm{Dp}_{1}(R)$ by [OY2, Lemma 2]. Since $\alpha$ is super-primitive over $R$, we see that $\operatorname{grade}\left(J_{[\alpha]}\right)>1$. Hence we have $\operatorname{grade}\left(\tilde{J}_{[\alpha]}\right)>1$, that is, $\tilde{J}_{[\alpha]_{p}}=R_{p}$ for every $p \in \mathrm{Dp}_{1}(R)$. So we conclude that $\alpha$ is exclusive over $R$ by Lemma 2.2.

Conversely, the set of diffusing points (i.e., $p \in \operatorname{Spec}(R)$ such that $p R[\alpha]_{p}=$ $\left.R[\alpha]_{p}\right)$ is given by $\bigcap_{i=1}^{d-1} V\left(I_{[\alpha]} \eta_{i}\right) \backslash V\left(I_{[\alpha]} \eta_{d}\right)=V\left(\tilde{J}_{[\alpha]}\right) \backslash V\left(I_{[\alpha]} \eta_{d}\right)$ by [OY2, Lemma 2]. So take $p \in \operatorname{Dp}_{1}(R)$ such that $p R[\alpha]_{p}=R[\alpha]_{p}$. Then $p \supseteq \tilde{J}_{[\alpha]}$ and $p \nsupseteq I_{[\alpha]} \eta_{d}$. Thus we have grade $\left(\tilde{J}_{[\alpha]}\right)=1$, which yields that $\alpha$ is not exclusive over $R$ by Lemma 2.2. Hence $q R[\alpha]_{q} \neq R[\alpha]_{q}$ for all $q \in \operatorname{I} m(\phi)$.

Proposition 2.6. Assume that $\alpha_{1}, \ldots, \alpha_{n}$ are super-primitive elements over $R$ and that $R$ contains a field. Put $A:=R\left[\alpha_{1}, \ldots, \alpha_{n}\right]$ and let $\phi: \operatorname{Spec}(A)$ $\rightarrow \operatorname{Spec}(R)$ be the canonical map induced from the inclusion $R \subseteq A$. If $\mathrm{Dp}_{1}(R)$ is contained in the image of $\phi$, then each $\alpha_{i}(1 \leq i \leq n)$ is exclusive over $R$.

Proof. Let $\psi_{i}: \operatorname{Spec}(A) \rightarrow \operatorname{Spec}\left(R\left[\alpha_{i}\right]\right)$ and $\phi_{i}: \operatorname{Spec}\left(R\left[\alpha_{i}\right]\right) \rightarrow \operatorname{Spec}(R)$ be the canonical maps induced from the inclusion $R\left[\alpha_{i}\right] \subseteq A$ and $R \subseteq R\left[\alpha_{i}\right]$, respectively. Then $\phi=\phi_{i} \cdot \psi_{i}$ induces the inclusions $\operatorname{Im}\left(\phi_{i}\right) \supseteq \operatorname{Im}(\phi) \supseteq \operatorname{Dp}_{1}(R)$. Thus our conclusion follows Theorem 2.5.

Problem. Is the converse statement of Proposition 2.6 valid?

Proposition 2.7. Assume that $\alpha$ is a super-primitive element of degree $d$ over $R$. If $\eta_{d} \in R$, then the canonical map $\phi: \operatorname{Spec}(R[\alpha]) \rightarrow \operatorname{Spec}(R)$ is surjective.

Proof. Note that $\phi: \operatorname{Spec}(R[\alpha]) \rightarrow \operatorname{Spec}(R)$ is surjective if and only if $V\left(\vec{J}_{[\alpha]}\right)=V\left(J_{[\alpha]}\right) \subseteq V\left(I_{[\alpha]} \eta_{d}\right)(c f$. [KY1, Theorem 7], [OY2,Lemma 2]). Since $\eta_{d} \in R$, we have $\eta_{d} I_{[\alpha]} \subseteq I_{[\alpha]}$, which implies that $V\left(\tilde{J}_{[\alpha]}\right)=V\left(J_{[\alpha]}\right)$ by the construction of $J_{[\alpha]}$. So we have our conclusion.

Note that $\phi: \operatorname{Spec}(R[\alpha]) \rightarrow \operatorname{Spec}(R)$ is surjective and $\phi$ is flat if and only if $\phi$ is faithfully flat. So we obtain the following corollary.

Corollary 2.8. Assume that $\eta_{d} \in R$. If $R[\alpha]$ is flat over $R$, then $R[\alpha]$ is faithfully flat over $R$.

The following proposition gives rise to a condition for $\eta_{d} \in R$. 
Proposition 2.9. Assume that $\alpha$ is a super-primitive element of degree $d$ over $R$ and that $R$ contains a field. Then the following statement are equivalent

(i) $\eta_{d} \in R$;

(ii) $\alpha$ is exclusive over $R$ and $I_{\eta_{d}} R[\alpha]=R[\alpha]$.

Proof. (i) $\Rightarrow$ (ii) follows from Lemma 2.2 .

(ii) $\Rightarrow$ (i) : Since $\eta_{d} \in I_{\eta_{d}}^{-1} R[\alpha]=I_{\eta_{d}}^{-1} I_{\eta_{d}} R[\alpha] \subseteq R[\alpha]$, we conclude that $\eta_{d} \in R[\alpha] \cap K=R$.

REMARK 2.10. Let $I$ denote an ideal of $R$. Then

$$
\operatorname{grade}(I)>1 \Leftrightarrow I^{-1}=R
$$

where $I^{-1}:=R: K I$.

THEOREM 2.11. Assume that $\alpha$ is an anti-integral element of degree d over $R$. Let $f: \operatorname{Spec}(R[\alpha]) \rightarrow \operatorname{Spec}(R)$ and $g: \operatorname{Spec}\left(R\left[\eta_{d}\right]\right) \rightarrow \operatorname{Spec}(R)$ be the canonical maps obtained from the inclusions $R \subseteq R[\alpha]$ and $R \subseteq R\left[\eta_{d}\right]$, respectively. If $\operatorname{grade}_{R[\alpha]}\left(J_{[\alpha]} R[\alpha]\right)>1, \operatorname{grade}_{R[\alpha]}\left(J_{\eta_{d}} R[\alpha]\right)>1$ and $\operatorname{grade}_{R\left[\eta_{d}\right]}\left(J_{[\alpha]} R\left[\eta_{d}\right]\right)>1$, then $R[\alpha] \cap K=R\left[\eta_{d}\right]$ and $\operatorname{I} m(f)=\operatorname{I} m(g)$.

Proof. First note that $J_{[\alpha]} R\left[\eta_{d}\right] \subseteq J_{[\alpha]}^{R\left[\eta_{d}\right]}$ (here we use the notation as in Remark 2.1; put $\left.S=R\left[\eta_{d}\right]\right)$. So $\operatorname{grade}_{R\left[\eta_{d}\right]}\left(J_{[\alpha]} R\left[\eta_{d}\right]\right)>1$ yields that $\operatorname{grade}_{R\left[\eta_{d}\right]}\left(J_{[\alpha]}^{R\left[\eta_{d}\right]}\right)>1$, so that $\alpha$ is super-primitive over $R\left[\eta_{d}\right]$. Take $P \in$ $\operatorname{Dp}_{1}(R[\alpha])$ and put $p:=P \cap R$. Since $J_{[\alpha]} R[\alpha] \nsubseteq P$, we have either $J_{[\alpha]} \nsubseteq p$ or $I_{\eta_{d}} \nsubseteq p$. Thus $R_{p}[\alpha]$ is flat over $R_{p}$ ( $c f$. [OSY]). So we have $I_{\eta_{d}} R_{p}[\alpha]=\left(R:_{R}\right.$ $\left.\eta_{d}\right) R_{p}[\alpha]=R_{p}[\alpha]:_{R_{p}[\alpha]} \eta_{d}=R_{p}[\alpha]$. Thus $\eta_{d} \in R_{p}[\alpha]$ by Proposition 1.1. Therefore $\eta_{d} \in \bigcap_{\left.P \in \mathrm{Dp}_{1}(R \mid \alpha]\right)} R[\alpha]_{P}=R[\alpha]$. As mentioned above, $\alpha$ is super-primitive over $R\left[\eta_{d}\right]$. Since $\eta_{d} \in R\left[\eta_{d}\right], \alpha$ is exclusive over $R\left[\eta_{d}\right]$. Hence the canonical map $\psi: \operatorname{Spec}(R[\alpha]) \rightarrow \operatorname{Spec}\left(R\left[\eta_{d}\right]\right)$ is surjective by Proposition 2.7. Consider the following commutative diagram :

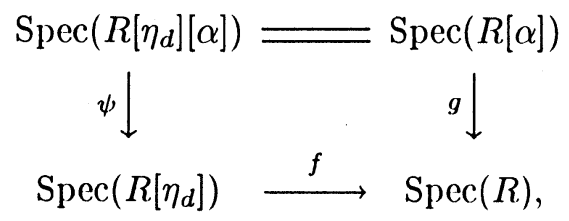

here we use that $\eta_{d} \in R[\alpha]$. Since $\psi$ is surjective and $f \cdot \psi=g$, we conclude that $\operatorname{Im}(f)=\operatorname{I} m(g)$.

We say that $\alpha$ is an ultra-primitive element of degree $d$ over $R$ if grade $\left(I_{[\alpha]}+\right.$ $\mathcal{C}(\bar{R} / R))>1$, where $\bar{R}$ denotes the integral closure of $R$ in $K$ and $\mathcal{C}(\bar{R} / R)$ denotes the conductor between $R$ and $\bar{R}$ (cf. [OY3]).

Proposition 2.12. Assume that an ultra-primitive element of degree $d$. If $\operatorname{grade}\left(I_{[\alpha]}: R I_{\left[\alpha^{-1}\right]}\right)>1$, then $\eta_{d} \in R$.

Proof. Take $p \in \mathrm{Dp}_{1}(R)$. Then either $I_{[\alpha]} \nsubseteq p$ or $\mathcal{C}(\bar{R} / R) \nsubseteq p$. If $I_{[\alpha]} \nsubseteq p$, then $I_{[\alpha]} \subseteq I_{\eta_{d}} \nsubseteq p$, that is, $\eta_{d} \in R_{p}$. If $I_{[\alpha]} \subseteq p$, then $\mathcal{C}(\bar{R} / R) \nsubseteq p$ and hence 
$R_{p}$ is a normal domain. Note that $I_{\left[\alpha^{-1}\right] p}=\eta_{d} I_{[\alpha] p} \subseteq I_{[\alpha] p}$ (cf. Lemma 1.3). The latter inclusion $\eta_{d} I_{[\alpha] p} \subseteq I_{[\alpha] p}$, that is, $\eta_{d} \in\left(I_{[\alpha]}: K I_{[\alpha]}\right)_{p}$ implies that $\eta_{d}$ is integral over $R_{p}$, noting that $I_{[\alpha] p}$ is finitely generated over $R_{p}$. So we have $\eta_{d} \in R_{p}$. Therefore $\eta_{d} \in \bigcap_{p \in \mathrm{Dp}_{1}(R)} R_{p}=R$.

\section{References}

[KY1] M.Kancmitsu and K.Yoshida, Anti-integral extensions and unramified extensions, Math. J. Okayama Univ. 36 (1994), 51-62.

[KY2] M.Kanemitsu and K.Yoshida, Some properties of extensions $R[\alpha] \cap R\left[\alpha^{-1}\right]$ over Noetherian domains $R$, Comm. in Alge. 23 (1995), 4501-4507.

[M] H.Matsumura, Commutative Algebra (2nd ed.), Benjamin, New York, 1980.

[OY1] S.Oda and K.Yoshida, Anti-integral extensions of Noetherian domains, Kobe J. Math. 5 (1988), 43-56.

[OY2] S.Oda and K.Yoshida, Remarks on an exclusive extension gencrated by a super-primitive element, Osaka J. Math. 32 (1995), 495-499.

[OY3] S.Oda and K.Yoshida, The behaviour of generalized denominator ideals in anti-integral extensions, Math. J. Ibaraki Univ. 29 (1997), 1-7.

[OY4] S.Oda and K.Yoshida, Super-primitive elements and ultra-primitive elements, to appear in Bull. Okayama Univ. of Sci..

[OSY] S.Oda, J.Sato and K.Yoshida, High degree anti-integral extensions of Noetherian domains, Osaka J. Math. 30 (1993), 119-135.

[YSO] K.Yoshida, J.Sato and S.Oda, On exclusive extensions of Noctherian domains, Bull. Okayama Univ. of Sci. 29 (1993), 21-26. 